from 2011 to 2014 was -0.57 percentage points while the average change in dialysis facility waitlisting rates from 2014 to 2017 was -4.17 percentage points. Half of facilities in the 2011 lowest quartile remained in the lowest quartile in $2017 ; 45 \%$ of facilities in the top 2011 quartile dropped into a lower quartile. The middle 2 quartiles were fairly evenly split between worsening, improving, and not changing. DISCUSSION/SIGNIFICANCE OF IMPACT: Average waitlisting rates have declined since KAS implementation. Many facilities switched quartiles from 2011-17 suggesting that facility waitlisting rates are unstable over time. The decline in waitlisting rates post-KAS suggests that new allocation rules may be discouraging patients and/or providers from getting ESRD patients waitlisted.

\section{The Role of Suggestibility and Trait Anxiety in Young Adult Alcohol Use}

Alexandra Cowand ${ }^{1}$, Melanie Schwandt ${ }^{2}$, Alyssa Schneider ${ }^{3}$, Jodi M. Gilman $^{4}$, Nancy Diazgranados ${ }^{2}$, Vijay A. Ramchandani ${ }^{2}$, and Bethany L. Stangl $\left.\right|^{2}$

${ }^{1}$ National Institutes of Health; ${ }^{2}$ National Institute on Alcohol Abuse and Alcoholism; ${ }^{3}$ Yale University; ${ }^{4}$ Harvard Medical School

OBJECTIVES/GOALS: The purpose of this study was to investigate how suggestibility and social susceptibility relate to alcohol use in young adult non-dependent alcohol users, and the role of trait anxiety in this relationship. We hypothesized that greater trait anxiety would be associated with higher levels of alcohol misuse, and this would be mediated by suggestibility. METHODS/STUDY POPULATION: Study participants enrolled in the NIAAA screening and assessment protocol completed questionnaires on suggestibility, anxiety, and alcohol use. The Multidimensional Iowa Suggestibility Scale (MISS) is a 95-question self-report assessment of suggestibility. Trait anxiety is assessed with the State Trait Anxiety Inventory-Trait (STAI-T). Alcohol measures included the Alcohol Use Disorder Identification Test (AUDIT). Structured Clinical Interviews for DSM-IV or DSM-5 disorders were conducted, and non-dependent participants $(\mathrm{N}=113)$ were considered. A median split was conducted (median age $=35.1$ years), with the focus of this study on the younger individuals $(\mathrm{N}=55)$. RESULTS/ANTICIPATED RESULTS: Initial analyses showed that suggestibility, alcohol misuse, and trait anxiety all had significant positive correlations with one another. To better understand the relationship of peer influence, specifically, with drinking and anxiety, MISS subscale of Peer Conformity was analyzed. MISS total score and Peer Conformity were positively correlated with AUDIT Total as well as STAI-T Score. STAI-T Score was additionally positively correlated with AUDIT Total $\left(\right.$ all $\left.p^{2}=0.222\right)$. We also looked at Peer Conformity in place of MISS Total $\left(\mathrm{R}^{2}=0.213\right)$. In both models, only suggestibility measures were significant predictors of harmful alcohol use $(\mathrm{p}<0.01)$. DISCUSSION/SIGNIFICANCE OF IMPACT: In young social drinkers, there were significant positive associations between suggestibility, risky alcohol use, and trait anxiety. These results suggest that suggestibility may be a modifiable risk factor for risky alcohol consumption. Future directions include using mediation models to explore the associations between suggestibility, anxiety, and alcohol misuse.
4024

Theory and Scale Development for Cancer-Related SelfEfficacy in Partners of Breast Cancer Survivors

Andrea Cohee ${ }^{1}$, Claire Draucker ${ }^{1}$, Patrick Monahan ${ }^{2}$, and Victoria Champion $^{1}$

${ }^{1}$ Indiana University School of Nursing; ${ }^{2}$ Indiana University School of Medicine

OBJECTIVES/GOALS: Specific aims are to: (Qualitative aim) Develop a new measure of cancer-related self-efficacy in partners (BCSES-P) and obtain feedback on the items (Quantitative) Evaluate the psychometric properties of the BCSES-P including: dimensionality, factor analysis, and construct validity assessing the relationships posited METHODS/STUDY POPULATION: 2Phase Approach: 1) Item development and 1) Item testing Phase 1 Stage 1: Literature review to identify additional covariates Stage 2: Focus groups and individual interviews to determine partners' needs Sample size: 20 partners (18 years of age or older, identifying as being in a committed relationship with a BCS) Design: cross-sectional, qualitative interviews Stage 3: Develop candidate items Stage 4: Cognitive interviews Stage 5: Finalize items with research team Phase 2 Preliminary psychometric testing Dimensionality Internal consistency reliability Construct validity Sample size: 150 partners Design: cross-sectional, online survey RESULTS/ANTICIPATED RESULTS: The BCSES-P will be unidimensional as assessed by exploratory factor analysis. The BCSES-P will demonstrate an internal consistency coefficient of 0.70 or above. Construct validity of the BCSES-P will be demonstrated by support of the following theoretical relationships: Cancer-related self-efficacy will be positively related to marital satisfaction and sexual functioning (social wellbeing) and the distal outcome, overall QoL. Cancer-related self-efficacy will be negatively related to fatigue (physical well-being), fear of recurrence, depression, and anxiety (psychological well-being). DISCUSSION/SIGNIFICANCE OF IMPACT: Findings will guide intervention development to improve partners' quality of life The BCSES-P will be the first scale to measure partners' cancer-related self-efficacy. This study will highlight a holistic approach to studying the long-term effects of breast cancer on partners.

4112

Tools for Self-Management of Obstetric Fistula in Low and Middle-income Countries: A Qualitative Study Exploring Pre-implementation Barriers and Facilitators among Global Stakeholders

Nessa E Ryan ${ }^{1}$, Bernadette Boden-Albala ${ }^{2}$, Gabriel Ganyaglo ${ }^{3}$, and Alison El Ayadi

${ }^{1} \mathrm{NYU}-\mathrm{H}+\mathrm{H}$ Clinical and Translational Science Institute; ${ }^{2}$ University of California, Irvine; ${ }^{3}$ Korle Bu Teaching Hospital

OBJECTIVES/GOALS: Insertable devices (IDs) for obstetric fistula (OF) management are feasible, acceptable, but not accessible; implementation determinants in low and middle-income countries (LMICs) are unknown. Thus, the purpose of this study was to understand pre-adoption facilitators and barriers among global stakeholders for a therapeutic ID for OF in LMICs. METHODS/STUDY POPULATION: Stakeholders, including researchers $(\mathrm{n}=11)$, clinicians $(n=4)$, government officials $(n=2)$, and administrators $(n=4)$, were purposefully identified from various sectors involved 